\title{
Causes of mortality among tuberculosis and HIV co-infected patients in Chiang Rai, Northern Thailand
}

Pacharee Kantipong'

Kuniko Murakami²

Saiyud Moolphate ${ }^{3}$

Myo Nyein Aung ${ }^{4,5}$

Norio Yamada ${ }^{2}$

'Chiang Rai Prachanukroh Hospital, Thailand; 'Japan Research Institute of Tuberculosis, Japan Anti-Tuberculosis Association, Tokyo, Japan; ${ }^{3} \mathrm{~TB} /$ HIV Research Project, Chiang Rai, Thailand; ${ }^{4}$ Department of Public Health, Graduate School of Medicine, Juntendo University, Tokyo, Japan; ${ }^{5}$ Department of Epidemiology, University of Public Health, Yangon, Myanmar
Correspondence: Pacharee Kantipong Chiang Rai Prachanukroh Hospital, Chiang Rai Province,

Thailand 57000

Email pachareek@hotmail.com
This article was published in the following Dove Press journal:

HIVIAIDS - Research and Palliative Care

3 October 2012

Number of times this article has been viewed

Background: The case fatality rate in patients with tuberculosis (TB) associated with human immunodeficiency virus (HIV) has been particularly high in Chiang Rai, Northern Thailand. It was almost $50 \%$ before the introduction of antiretroviral therapy in the last decade, and was still at $28 \%$ in 2008 , despite expanding access to antiretroviral therapy. Reviewing the causes of death may lead to further understanding of the timeline and natural history of TB-HIV coinfection, and in so doing help to devise an effective prevention strategy in Chiang Rai. In this study, we aimed to investigate the distribution of confirmed causes of death in patients coinfected with TB and HIV in Chiang Rai, describe the causes of such deaths along the timeline of TB treatment, and identify predictors of each cause of death.

Methods: In this retrospective study, we reviewed the causes of death for 331 patients who died of TB-HIV coinfection at Chiang Rai Prachanukroh Hospital from 2005 to 2008. Causes of death were confirmed by reviewing medical records, vital registration, and the TB register in the province, as well as obtaining reconfirmation by two experienced HIV physicians.

Results: The confirmed causes of death were TB (39\%), acquired immune deficiency syndrome (AIDS)-related opportunistic infections other than TB (AOI) (29\%), and other systemic diseases which were neither TB nor AIDS-related opportunistic infections (nonTB-nonAOI) $(16 \%)$. The definitive cause could not be confirmed in the remaining $16 \%$ of deaths. After starting the TB treatment, deaths caused by TB occurred earlier compared with deaths caused by AOI, which occurred steadily throughout the course of TB treatment, whilst deaths caused by non-TB-nonAOI increased gradually in later months. Further analysis by multivariate multinomial regression analysis showed that deaths in the first month (adjusted odds ratio [aOR] 4.64, 95\% confidence interval [CI] 2.49-8.63), CD4 count $\geq 200$ cells $/ \mathrm{mm}^{3}$ (aOR 5.33, CI 1.05-26.10), non-category 1 TB treatment regimens (aOR 5.23, CI 1.04-9.77), and TB meningitis (aOR 3.27, CI 1.37-7.82) were significant predictors of confirmed TB deaths. Moreover, age over 45 years (aOR 3, CI 1.32-6.84) and admission as an inpatient were predictors of death caused by neither TB nor AIDS-related opportunistic infections (aOR 3.08, CI 1.39-6.80). Additional analysis showed that non-Thai patients (aOR 0.35, CI 0.12-0.99), those with an unknown CD4 count at TB diagnosis (aOR 0.16, CI 0.08-0.33), and those without an HIV diagnosis before TB treatment (aOR 0.32, CI 0.18-0.59) were less able to access antiretroviral therapy.

Conclusion: The timeline and predictors of causes of death may assist in devising an intervention strategy for further reduction of the TB-HIV case fatality rate.

Keywords: human immunodeficiency virus, tuberculosis, coinfection, cause of death, CD4, antiretroviral therapy 


\section{Introduction}

Tuberculosis (TB), the leading cause of death in people living with human immunodeficiency virus (HIV), is a current global health challenge. ${ }^{1}$ Expanding use of antiretroviral therapy has decreased acquired immune deficiency syndrome (AIDS)related deaths in both developing and developed countries..$^{2-5}$ Nevertheless, TB consistently causes a large number of deaths among people living with HIV globally, especially in highburden settings, such as southeast Asia and Africa. ${ }^{6-9}$ Death review studies to find out the distribution of causes of death in TB-HIV coinfected patients are necessary to devise specific preventive interventions in each high-burden setting.

HIV and TB are interacting epidemic diseases. In 2009, there were an estimated 1.1 million HIV-infected patients with TB globally. ${ }^{10}$ Thailand, the country with the highest HIV prevalence in southeast Asia, has the 22nd highest TB burden in the world. According to recent estimates by the World Health Organization, $1.3 \%$ of Thai adults are HIV-infected and the TB prevalence is 189 per 100,000 population. ${ }^{11,12}$ The HIV epidemic has fuelled the TB burden in the last two decades. Concurrently, $16 \%$ of newly-diagnosed TB cases in Thailand were HIVinfected, ${ }^{12-14}$ whilst TB mortality in the HIV-positive population was estimated as six per 100,000 per year in 2007. ${ }^{13}$

Dual epidemics have affected Chiang Rai, the northernmost province of Thailand, bordering Myanmar and Laos. The HIV prevalence among TB patients in Chiang Rai was $41 \%$ in 2000 and $27 \%$ in $2010 .{ }^{15}$ It was relatively higher than the recent national figure of $16 \% \cdot{ }^{14}$ In the last decade of the growing HIV epidemic, the TB case fatality rate was particularly high in Chiang Rai at $56 \%$ in 2000 , falling to $23.4 \%$ in 2009 and rising again to $26.1 \%$ in $2010 .{ }^{15}$

People living with HIV in regions where TB prevalence is high are at a higher risk of Mycobacterium tuberculosis infection, often progressing to active TB disease, and in turn to rapid and severe immunological compromise. ${ }^{16}$ Thereafter, people living with HIV can die of TB as well as other AIDS-related opportunistic infections (AOI). Intolerance to multiple drug regimens and adverse drug interactions are other untoward effects of TB treatment that can lead to severe liver injury and death. ${ }^{17}$ According to World Health Organization TB treatment outcome definitions, TB control programs routinely count any death during TB treatment as "TB treatment outcome death". ${ }^{18}$ In fact, the definitive causes of such deaths, in the presence of concomitant HIV infection, are not totally confined to TB and thus warrant further investigation. Therefore, we conducted this study with the aim of investigating the definitive causes of death in patients coinfected with TB-HIV, and describing those causes of death within the timeline of TB treatment. Further, we attempted to identify predictors for each cause of death.

\section{Materials and methods Study setting and population}

This study was conducted at Chiang Rai Prachanukroh Hospital $(\mathrm{CPH})$ where the health insurance system covers treatment and health service costs of HIV and TB infection for all Thai citizens. The inclusion criteria were: (1)TB-HIV co-infected patients who were registered for TB treatment at $\mathrm{CPH}$ from October 2005 to September 2008 and died during TB treatment, (2) both sputum smear positive and negative TB, (3) both pulmonary and extra-pulmonary TB. We screened cases in the TB register by the TB treatment outcome of "death". We followed up every case from the time of TB diagnosis until death. The exclusion criterion was a lack of medical records to review the cause of death. There was a total of 361 eligible cases by inclusion criteria; however, 30 cases were without medical records and therefore excluded. The final analysis included 331 cases.

\section{Ethical approval}

This study was approved by the CPH Ethics Committee, Thailand, and the Ethical Review Board of the Research Institute of Tuberculosis, Japan. The ethics committee waived the need for informed consent because this was a retrospective study using routine hospital-based data. It did not inflict harm on any of the patients; rather, it was operationally necessary research with the aim of reducing TB-HIV mortality in the region. Personal patient details were kept strictly secure and confidential by recoding the case number. There were no personal data stored in the database created. The authors accessed the database by password and signed a clause preventing breach of confidentiality.

\section{Causes of death}

Two experienced HIV physicians reviewed the medical records, in the form of either inpatient or outpatient charts, to confirm the most likely cause of death. Vital status was confirmed by three registries, ie, provincial TB registers, medical records at $\mathrm{CPH}$, and Thailand's national online vital registry.

\section{Diagnosis of TB, HIV, and AIDS-related opportunistic infections}

TB was routinely diagnosed by the consistency of the clinical impression of the physician, chest $\mathrm{x}$-ray, and microscopic examination of the sputum smear or TB culture. We reconfirmed the certainty of all the TB diagnoses. We defined the cases as "bacteriologically positive TB" and "presumptive 
TB". Bacteriologically positive TB cases had a positive confirmatory test result, which was either a sputum smear positive for acid-fast bacilli or a positive TB culture. On the other hand, presumptive TB cases were diagnosed by consistent clinical features, chest $\mathrm{x}$-ray results, and the impression of the attending physician, albeit with a lack of either a positive smear acid-fast bacilli test or a positive culture result. HIV infection was diagnosed by the HIV antibody test using an enzyme-linked immunosorbent assay. Clinical diagnoses of opportunistic infection other than TB, eg, cryptoccocal meningitis or penicilliosis, were confirmed by available clinical laboratory tests at $\mathrm{CPH}$, a tertiary level hospital.

Antiretroviral therapy regimens recommended by Thailand National guidelines were used to treat HIV-infected patients with TB. ${ }^{19}$ These regimens followed the World Health Organization guidelines for national TB treatment programs. ${ }^{20}$

\section{Data management and analysis}

Well structured case record forms were used for reviewing the medical records and laboratory data. Microsoft Office Excel 2007 and Look-like were used for data collection and validation. Characteristics of patients coinfected with TB-HIV were summarized descriptively as categorical data by percentage, continuous data by the mean and standard deviation, or median, maximum, minimum, or interquartile range based on normality. Furthermore, our analysis sought factors predicting each cause of death category using multinomial logistic regression analysis and factors predicting access to antiretroviral therapy using binomial logistic regression analysis. Univariate analysis predicting causes of death included all logically suitable variables, ie, time of death, age group, gender, race, education, occupation, inpatient or outpatient status, type of TB regimen, TB sites, CD4 count in category, use of antiretroviral therapy, preventive cotrimoxazole and fluconazole therapy, and time of HIV diagnosis. Univariate analysis for predicting access to antiretroviral therapy included age, gender, race, CD4 count category, and time of HIV test compared with TB diagnosis. Variables with a $P$ value $<0.2$ in univariate analysis were included in the multivariate analysis models. Stata version 11 (Stata Corporation, College Station, TX) was used for data analysis. Statistical significance was considered as a $P$ value $<0.05$ with a $95 \%$ confidence interval.

\section{Results}

The present study reviewed 331 persons who died of TB-HIV dual infection over three successive years. Median life expectancy after diagnosis of TB was 50 (interquartile range 16-116) days and was less variable through the study period. Median age of death was 35 (interquartile range 30-42) years. Overall, the proportion of male deaths was double that of female deaths (66\% versus 33\%). The majority of patients were Thai $(89.73 \%)$ and the rest were migrants or from the hill tribes. Most of the study population were casual laborers $(43.75 \%)$ or farmers $(26.25 \%)$. Furthermore, 121 of the 331 cases $(36.56 \%)$ died within 30 days of starting TB treatment. Table 1 shows the details of the characteristics of the study population.

\section{Causes of death in patients with TB/HIV at CPH 2005-2008}

After reconfirmation, the primary causes of death among the 331 persons were determined as follows: TB, 130 (39.3\%); AOI, 94 (28.4\%); nonTB/nonAOI, systemic diseases (such as renal or liver disease), 54 (16.3\%); and ambiguous causes, 53 (16\%, Table 1). Among the TB deaths, $36.9 \%$ were caused by pulmonary TB, and the rest were either extra-pulmonary TB or both extra-pulmonary and pulmonary TB. One fourth of the deaths were caused by TB meningitis. Among AOI, Cryptococcus meningitis (25.5\%) and Pneumocystis jiroveci pneumonia (PCP ) (20.2\%) were common. Among the nonTB/nonAOI systemic causes, sepsis (35.2\%) and cirrhosis (20.4\%) were the most common conditions (Table 2).

\section{Cause of death along the timeline of TB treatment}

The distribution of causes of death among patients coinfected with TB-HIV is displayed along the timeline of TB treatment in Figure 1. Of 331 patients, 121 died in the first month (36.7\%) and 62 died in the second month (18.7\%). As can be seen clearly, most of the TB deaths occurred within the first month of the start of TB treatment. Among the 121 deaths in the first month, 81 (66.9\%) were confirmed as due to TB, with $37.1 \%$ attributable to TB meningitis. Deaths caused by AOI occurred constantly throughout the timeline of TB treatment, with a peak in the fourth month. Systemic diseases caused increasing numbers of deaths in the later months of TB treatment.

Multinomial regression analysis identified factors associated with each outcome category of cause of death (Table 3 ). Death in the first month (aOR 4.64, CI 2.49-8.63), TB meningitis (aOR 3.27, CI 1.37-7.82), a non-category 1 regimen (aOR 3.19, 1.04-9.77), and a CD4 count $\geq 200$ cells $/ \mathrm{mm}^{3}$ at TB diagnosis (aOR 5.23, CI 1.05-26.10) were significantly associated with TB deaths.

Conversely, there was a significant negative association between the occupational groups "casual laborers and 
Table I Characteristics of deceased TB-HIV coinfected patients, Chiang Rai Prachanukroh Hospital, Northern Thailand, 2005-2008

\begin{tabular}{|c|c|c|}
\hline Characteristics & $\mathbf{n}$ & $\%$ \\
\hline Total number & 331 & 100 \\
\hline \multicolumn{3}{|l|}{ Gender } \\
\hline Male & 219 & 66.2 \\
\hline Female & 112 & 33.8 \\
\hline \multicolumn{3}{|l|}{ Age (years) } \\
\hline$<45$ & 263 & 79.5 \\
\hline$\geq 45$ & 68 & 20.5 \\
\hline Median & 35 & \\
\hline Range & $30-42$ & \\
\hline \multicolumn{3}{|l|}{ Ethnicity } \\
\hline Thai & 297 & 89.7 \\
\hline Non-Thai ${ }^{\$}$ & 34 & 10.3 \\
\hline \multicolumn{3}{|l|}{ Marital status } \\
\hline Married & 146 & 44.5 \\
\hline Single & 87 & 26.5 \\
\hline Widowed/divorced & 95 & 29.0 \\
\hline \multicolumn{3}{|l|}{ Education } \\
\hline Uneducated & 24 & 10.2 \\
\hline Primary & 150 & 63.8 \\
\hline Secondary & 55 & 23.4 \\
\hline Higher than secondary & 6 & 2.6 \\
\hline \multicolumn{3}{|l|}{ Occupation } \\
\hline Casual laborer & 140 & 43.8 \\
\hline Farmer & 84 & 26.3 \\
\hline Household worker & 36 & 11.3 \\
\hline Prisoner & 16 & 5.0 \\
\hline Business owner & 13 & 4.1 \\
\hline Government employee & 6 & 1.9 \\
\hline Other & 6 & 1.9 \\
\hline Unemployed & 19 & 5.9 \\
\hline \multicolumn{3}{|l|}{ TB-related information } \\
\hline \multicolumn{3}{|l|}{ Site of TB } \\
\hline Pulmonary TB & 134 & 40.5 \\
\hline Extrapulmonary with pulmonary TB & 64 & 19.3 \\
\hline Extrapulmonary TB & 133 & 40.2 \\
\hline Location/type of extrapulmonary TB & 197 & \\
\hline Meningeal & 60 & 30.5 \\
\hline Lymphatic & $4 \mid$ & 20.8 \\
\hline Intestinal & 38 & 19.3 \\
\hline Disseminated & 36 & 18.3 \\
\hline Pleural & 12 & 6.1 \\
\hline Bone & 4 & 2.0 \\
\hline Liver & 4 & 2.0 \\
\hline Pericardial & 1 & 0.5 \\
\hline Cutaneous & 1 & 0.5 \\
\hline \multicolumn{3}{|l|}{ TB treatment regimen } \\
\hline CAT-I regimen & 301 & 91.0 \\
\hline Non-CAT-I regimens & 30 & 9.0 \\
\hline \multicolumn{3}{|l|}{ Tuberculosis diagnosis reconfirmation* } \\
\hline Definite & 102 & 30.8 \\
\hline Probable & 46 & 13.9 \\
\hline Presumptive & 165 & 49.9 \\
\hline NTM & 18 & 5.4 \\
\hline \multicolumn{3}{|l|}{ MDR TB } \\
\hline Yes & 7 & 2.1 \\
\hline No & 324 & 97.9 \\
\hline
\end{tabular}

Table I (Continued)

\begin{tabular}{|c|c|c|}
\hline Characteristics & $\mathbf{n}$ & $\%$ \\
\hline Total number & 331 & 100 \\
\hline \multicolumn{3}{|l|}{ HIV-related information } \\
\hline \multicolumn{3}{|l|}{ Time of HIV diagnosis } \\
\hline Known before TB treatment & 219 & 66.2 \\
\hline Not known before TB treatment & 112 & 33.8 \\
\hline \multicolumn{3}{|l|}{ CD4 count (cells $\left./ \mathrm{mm}^{3}\right)$} \\
\hline CD4 count $<200$ & 205 & 61.93 \\
\hline CD4 count $\geq 200$ & 23 & 6.95 \\
\hline Unknown & 103 & 31.12 \\
\hline Median CD4 count & 37 & \\
\hline Range & $14-124$ & \\
\hline \multicolumn{3}{|l|}{ ART status } \\
\hline Received after TB diagnosis & 35 & 10.6 \\
\hline Received before TB diagnosis & 73 & 22.1 \\
\hline Not received & 223 & 67.4 \\
\hline ART regimen (last regimen during TB treatment) & 108 & \\
\hline NVP base & 77 & 71.3 \\
\hline EFV base & 24 & 22.2 \\
\hline PI base & 4 & 3.7 \\
\hline Other & I & 1.0 \\
\hline Unknown & 2 & 1.8 \\
\hline \multicolumn{3}{|l|}{ Preventive cotrimoxazole therapy } \\
\hline Yes & 224 & 67.7 \\
\hline No & 107 & 32.3 \\
\hline \multicolumn{3}{|l|}{ Preventive fluconazole therapy } \\
\hline Yes & 175 & 52.9 \\
\hline No & 156 & 47.1 \\
\hline \multicolumn{3}{|l|}{ Time of death after start of TB treatment } \\
\hline Within 30 days & 121 & 36.56 \\
\hline Later than 30 days & 210 & 63.44 \\
\hline
\end{tabular}

Notes: Definitive: evidence for TB diagnosis from both positive smear for acidfast bacilli and culture growth for Mycobacterium tuberculosis. Probable: evidence for TB diagnosis from positive smear for acid-fast bacilli but no culture confirmation. Presumptive: neither smear acid-fast bacilli positive nor culture confirmation.

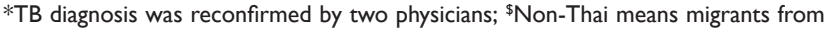
Laos and Myanmar and hill tribes.

Abbreviations: ART, antiretroviral therapy; NTM, nontuberculous mycobacterium; MDR TB, multidrug resistant tuberculosis; TAD, treatment after default; EFV, efavirenz; NVP, nevirapine; Pl, protease inhibitor; HIV, human immunodeficiency virus; CAT-I, World Health Organization standard category I TB treatment regimen (2HRZE/4HR).

farmers" and TB deaths (aOR 0.49, CI 0.19-1.25). Thus, these occupation groups were positively associated with the base outcome, ie, deaths from AOI. In addition, those older than 45 years (aOR 3, CI 1.32-6.84) and admitted as inpatients (aOR 3.8, CI 1.39-6.80) were more likely to die of systemic causes not attributable to TB or AOI.

\section{Indicators of HIV care in study population}

One third of the patients did not receive CD4 count testing(Table 1). The median CD4 count in known cases was $37 \mathrm{cells} / \mathrm{mm}^{3}$. More than half $(66.2 \%)$ of the study group knew their HIV status before their TB diagnosis. At the time of TB 


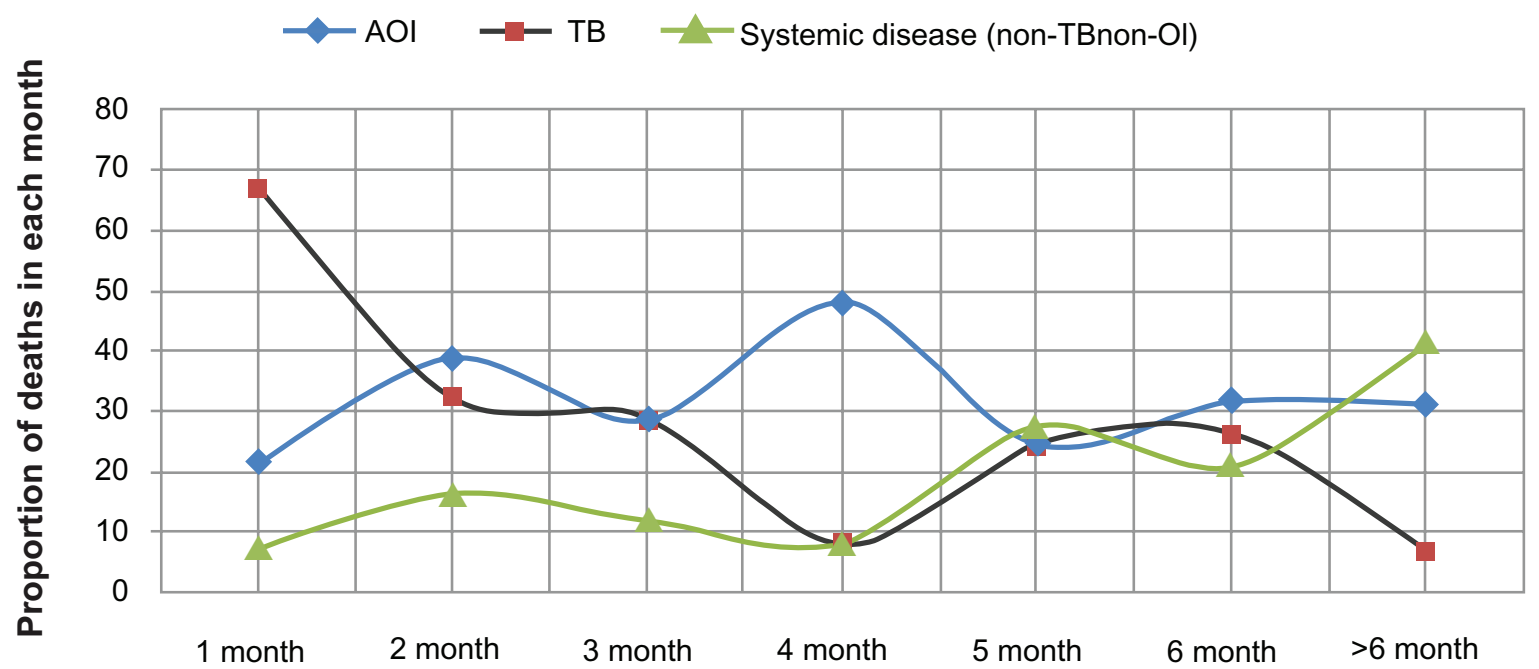

Figure I Causes of death shown by proportion of deaths in each month of TB treatment $(n=33 I, P<0.00 I)$.

Note: The proportion of undefined causes is not shown.

Abbreviations: AOI, AIDS-related opportunistic infection other than TB; TB, tuberculosis.

diagnosis, $22.1 \%$ of the patients were receiving antiretroviral therapy, $67.7 \%$ were on preventive cotrimoxazole therapy and $52.9 \%$ were on preventive fluconazole therapy. Further analysis by multivariate binomial logistic regression identified factors predicting access to antiretroviral therapy. Lack of antiretroviral therapy was significantly predicted by being
non-Thai, having an unknown CD4 count at TB diagnosis, and not having had an HIV test before TB diagnosis (Table 4).

\section{Discussion}

TB is a consistently lethal disease and the leading cause of morbidity and mortality for people living with HIV in the

Table 2 Reconfirmed details of causes of death of 33I persons with TB-HIV coinfection, Chiang Rai, Thailand, 2005-2008

\begin{tabular}{|c|c|c|c|c|c|}
\hline Confirmed cause of death & $\mathbf{n}$ & $\%$ & Detailed cause of death & $\mathbf{n}$ & $\%$ \\
\hline \multirow[t]{6}{*}{ Tuberculosis } & 130 & 39.27 & Pulmonary TB & 48 & 36.9 \\
\hline & & & Meningeal TB & 32 & 24.6 \\
\hline & & & Disseminated TB & 14 & 10.8 \\
\hline & & & Intra-abdominal TB & 16 & 12.3 \\
\hline & & & Lymphatic TB & 13 & 10.0 \\
\hline & & & Other TB & 7 & 5.38 \\
\hline \multirow[t]{9}{*}{ AIDS-related opportunistic infections } & 94 & 28.40 & Cryptococcus meningitis & 24 & 25.5 \\
\hline & & & $\mathrm{PJP}$ & 19 & 20.2 \\
\hline & & & NTM & 17 & 18.1 \\
\hline & & & Toxoplasmosis & 11 & 11.7 \\
\hline & & & Penicilliosis & 4 & 4.3 \\
\hline & & & Lymphoma & 2 & 2.1 \\
\hline & & & Chronic diarrhea & 2 & 2.1 \\
\hline & & & Other & 10 & 10.6 \\
\hline & & & HIV-related nonspecific & 5 & 5.3 \\
\hline NonTB -nonAIDS-related opportunistic & 54 & 16.31 & Sepsis & 19 & 35.2 \\
\hline \multirow[t]{5}{*}{ infections (or) systemic causes } & & & Cirrhosis & 11 & 20.4 \\
\hline & & & Renal insufficiency & 7 & 13.0 \\
\hline & & & Suicide & 5 & 9.3 \\
\hline & & & Gastrointestinal hemorrhage & 3 & 5.6 \\
\hline & & & Other & 9 & 16.7 \\
\hline
\end{tabular}

$16 \%$

Notes: Among the TB deaths, $36.9 \%$ were caused by pulmonary TB, and the rest were either extrapulmonary TB or both extrapulmonary and pulmonary TB. One fourth of the deaths were caused by TB meningitis. Among AIDS-related opportunistic infections, cryptococcal meningitis (25.5\%) and Pneumocystis jiroveci pneumonia (20.2\%) were common. Among the systemic causes not related to nonTB-nonAIDS-related opportunistic infections, sepsis (35.2\%) and cirrhosis (20.4\%) were the most common conditions.

Abbreviations: AIDS, acquired immunodeficiency syndrome; AOI, AIDS-associated opportunistic infections; NTM, nontuberculous mycobacterium; PJP, Pneumocystis jiroveci pneumonia; TB, tuberculosis. 
Table 3 Factors associated with the causes of TB-HIV coinfected patients' deaths in Chiang Rai, Thailand, 2005-2008

\begin{tabular}{|c|c|c|c|c|c|c|}
\hline \multirow{2}{*}{$\begin{array}{l}\text { Cause of death } \\
\text { Risk factors }\end{array}$} & \multicolumn{3}{|l|}{ TB } & \multicolumn{3}{|c|}{ NonTB/nonAOI } \\
\hline & aOR & $95 \% \mathrm{Cl}$ & $P$ value & aOR & $95 \% \mathrm{Cl}$ & $P$ value \\
\hline Death in the first month & 4.64 & $2.49-8.63$ & $<0.001$ & 0.46 & $0.19-1.14$ & 0.09 \\
\hline Death after the first month & I & & & 1 & & \\
\hline Age $\geq 45$ years & 1.01 & $0.46-2.23$ & 0.98 & 3 & $1.32-6.84$ & 0.01 \\
\hline Age $<45$ years & 1 & & & & & \\
\hline Non-Thai & 1.48 & $0.55-3.95$ & 0.44 & 2.09 & $0.59-7.4 I$ & 0.25 \\
\hline Thai & 1 & & & 1 & & \\
\hline Casual laborer and farmer & 0.43 & $0.19-0.94$ & 0.03 & 0.49 & $0.19-1.25$ & 0.14 \\
\hline Other occupations & 1 & & & 1 & & \\
\hline Inpatient & 0.75 & $0.4 I-1.36$ & 0.34 & 3.08 & I.39-6.80 & 0.01 \\
\hline Outpatient & 1 & & & 1 & & \\
\hline TB meningitis & 3.27 & I.37-7.82 & 0.01 & I.II & $0.37-3.30$ & 0.85 \\
\hline Other extrapulmonary TB & $1.4 \mathrm{I}$ & $0.73-2.72$ & 0.31 & 0.97 & $0.45-2.12$ & 0.95 \\
\hline Pulmonary TB & 1 & & & 1 & & \\
\hline Non-CAT-I regimens & 3.19 & I.04-9.77 & 0.04 & 1.37 & $0.32-5.82$ & 0.67 \\
\hline CAT-I regimen & I & & & 1 & & \\
\hline CD4 count $\geq 200$ at TB diagnosis & 5.23 & $1.05-26.10$ & 0.04 & 3.14 & $0.55-18.00$ & 0.19 \\
\hline CD4 count $<200$ at TB diagnosis & I & & & 1 & & \\
\hline
\end{tabular}

Note: Multinomial logistic regression analysis with base outcome AIDS-related opportunistic infections.

Abbreviations: AOI, AIDS-related opportunistic infections; aOR, adjusted odds ratio; CI, 95\% confidence interval; CD4 count, CD4 + T cells count by cells/mm³ CAT-I, World Health Organization standard category I TB treatment regimen (2HRZE/4HR); TB, tuberculosis.

developing world. ${ }^{21-24}$ The primary cause of death in patients coinfected with TB-HIV is not only TB, but also a range of other AOI. ${ }^{25}$ Thus, the TB deaths, crudely defined and counted according to the World Health Organization TB treatment outcome definition, were reviewed and further investigated in this study. ${ }^{15}$ To our knowledge, this study probably represents the most comprehensive review of the primary causes of death among persons with TB-HIV coinfection in southeast Asia, by observing a large sample of TB-HIV deaths over a long period of observation.

The current study exclusively observed people living with HIV, active TB, and advanced immunosuppression (median CD4 + T cells count 37 cells $/ \mathrm{mm}^{3}$ ) who died during

Table 4 Factors predicting access to antiretroviral therapy among deceased TB-HIV patients, Chiang Rai, Thailand 2005-2008

\begin{tabular}{lllll}
\hline \multirow{2}{*}{ Factors } & \multicolumn{4}{l}{ Antiretroviral therapy access } \\
\cline { 2 - 5 } & Prevalence \% & aOR & $\mathbf{9 5 \%} \mathbf{~ C l}$ & P value \\
\hline Non-Thai & 14.71 & 0.35 & $0.12-0.99$ & 0.047 \\
Thai & 34.68 & 1 & & \\
CD4 count unknown & 9.71 & 0.16 & $0.08-0.33$ & $<0.01$ \\
CD4 count $\geq 200$ & 47.83 & 1.21 & $0.49-2.98$ & 0.86 \\
CD4 count $<200$ & 42.44 & 1 & & \\
HIV test after & 16.07 & 0.32 & $0.18-0.59$ & $<0.01$ \\
TB diagnosis & & & & \\
HIV test before & 41.10 & 1 & & \\
TB diagnosis & & & & \\
\hline
\end{tabular}

Abbreviations: aOR, adjusted odds ratio; $\mathrm{Cl}$, confidence interval; $\mathrm{HIV}$, human immunodeficiency virus; $T B$, tuberculosis; $C D 4$ count, $C D+T$ cells $/ \mathrm{mm}^{3}$.
TB treatment. The age and gender distribution indicated that the TB-HIV deaths occurred mainly in young adults (median age 35 years) and predominantly among males. CD4 count data was lacking in $30 \%$ of the sample. Only one third of the sample received antiretroviral therapy.

\section{Timeline and predictors of TB-HIV deaths}

In the present study, TB represented the largest proportion of causes of death (39.27\%). We identified the characteristic predictors of these TB deaths (Table 3). TB meningitis, a lethal form of TB, was a significant predictor of death caused by TB (aOR 3.27, CI 1.37-7.83). A recent clinical trial in Vietnam reported a very high death rate caused by TB meningitis $(>55 \%)$, regardless of concomitant or deferred antiretroviral therapy, from 2 months after the beginning of TB treatment. ${ }^{26}$ Association with a non-category $1 \mathrm{~TB}$ regimen (aOR 3.19, CI 1.04-9.77) further suggested likely existence of drug resistance in retreated cases. We found that a CD4 count above 200 cells $/ \mathrm{mm}^{3}$ at TB diagnosis (aOR 5.23, CI 1.05-26.10) was a significant predictor of TB-related death, and possibly linked to immune reconstitution syndrome. ${ }^{27}$

Moreover, TB deaths were more frequently found within a month of starting TB treatment (Figure 1). This observation is confirmed by the result of multivariate analysis. Death in the first month was more likely to be caused by TB (aOR 4.64, CI 2.49-8.63, Table 3). This finding is consistent with the epidemiology of TB mortality reported by a 12-year surveillance 
study in Chiang Rai, ${ }^{28}$ and an autopsy-based death review study in Sao Paulo, Brazil, which reported an association between multibacillary and exudative response and TB-HIV deaths within 14 days of starting TB treatment. ${ }^{29}$

Overall, TB was the major cause of death, although it was not the primary cause of death in more than $50 \%$ of cases. This information indicates a need for multiple interventions to reduce the mortality among TB-HIV coinfected patients. In the present study, AOI were found to be the second leading cause of death. Furthermore, we identified that death among the occupational groups of farmers and laborers was less likely to be caused by TB (aOR 0.43 , CI 0.19-0.94) and more likely to be due to AOI, ie, the base outcome in the analysis. The distribution of deaths attributable to AOI was consistent throughout the course of TB treatment, with a peak in the fourth month. The most frequent AIDS-related opportunistic infection was cryptococcal meningitis. Cryptococcal infection has been reported as the second most common respiratory infection among people living with HIV in Thailand. ${ }^{30}$ The reason for prediction of deaths attributable to AOI in such occupational groups could be lack of awareness about preantiretroviral therapy care, as well as occupational exposure to environmental sources of opportunistic infection.

Cain et al reported a similar distribution of causes of death in TB-HIV patients. ${ }^{2}$ In a review of 142 deaths of patients coinfected with TB-HIV in central and northeastern parts of Thailand, they found that the highest proportion of deaths was due to AOI (45\%), with the second highest due to TB (27\%). The authors suspected ineffectiveness of preventive cotrimoxazole therapy. That study and ours differ slightly in terms of the immunological status of study population by of the median CD 4 count at TB diagnosis $\left(55\right.$ cells $/ \mathrm{mm}^{3}$ versus 37 cells $/ \mathrm{mm}^{3}$, respectively), and a higher TB incidence and case fatality rate in Chiang Rai. ${ }^{17}$ Moreover, early deaths, within 14 days of initiation of TB drugs, were excluded in the study reported by Cain et al. Such deaths, according to our analysis and a previous autopsy-based study, could be TB-related ${ }^{29}$ (Table 3).

Moreover, we found that death due to systemic diseases such as sepsis, cirrhosis, and renal failure, was predictable by age over 45 years (aOR 3.00, CI 1.32-6.84) and being admitted as inpatients (aOR 3.8, CI 1.39-6.8). Among the systemic causes of death, $35 \%$ were confirmed as sepsis and $20.4 \%$ as cirrhosis. The numbers of such deaths were not high in the initial months, but gradually increased towards the end of TB treatment.

\section{HIV care}

In our study, more than half of the deceased had received preventive cotrimoxazole and fluconazole therapy, whereas only one third of the deceased had received antiretroviral therapy. The median CD4 + T cells count of the study sample was 37 cells $/ \mathrm{mm}^{3}$, with $69 \%$ having a CD4 count of less than 200 cells $/ \mathrm{mm}^{3}$. According to current World Health Organization recommendations for antiretroviral therapy, all of the 331 patients merited antiretroviral therapy because their disease diagnosis was of active TB. ${ }^{31}$

During our study period (2005-2008), there was a global knowledge gap regarding the appropriate timing of antiretroviral treatment and TB therapy, weighing up the survival benefit against the risk of onset of immune reconstitution syndrome. ${ }^{27} \mathrm{Up}$ until 2008, Thai AIDS Society guidelines had recommended initiating antiretroviral therapy in people living with HIV and active TB disease at a CD4 count $<100$ cells $/ \mathrm{mm}^{3}$, as soon as the patient can tolerate anti-TB treatment. ${ }^{32}$ More recent 2010 Thai AIDS Society guidelines saw a change to the recommendations, instead beginning antiretroviral therapy at a CD4 count $<200$ cells $/ \mathrm{mm}^{3}$ in TB-HIV coinfection, 2-4 weeks after initiation of anti-TB therapy. ${ }^{33}$ These knowledge gaps, in the presence of a very high case fatality rate among HIV patients diagnosed with TB, could have made the physicians reluctant to start antiretroviral therapy.

In 2010 and thereafter, three clinical trials, ie, SAPIT (Starting Antiretroviral therapy at three Points In Tuberculosis therapy), CAMELIA (Cambodian Early versus Late Introduction of Antiretroviral Drugs), and STRIDE (Immediate Versus Deferred Start of Anti-HIV Therapy in HIV-Infected Adults Being Treated for Tuberculosis), conclusively confirmed that starting antiretroviral therapy within 2 weeks of initiation of TB therapy in patients with HIVassociated pulmonary TB and CD4 counts $<50$ cells $/ \mathrm{mm}^{3}$ is beneficial to reducing the mortality and incidence of new AIDS-defining illnesses. ${ }^{34-36} \mathrm{~A}$ logistical challenge in the developing world sees patients with suspected TB-HIV coinfection as needing to have their CD4 cell counts measured at the time of sputum microscopy. ${ }^{37}$ In our present study, 30\% of the deceased patients could not have their CD4 count measured until death.

Thus, we quantified the barriers of access to antiretroviral therapy by multivariate analysis (Table 4). Non-Thai ethnicity (aOR 0.35 , CI $0.12-0.99$ ), lack of a CD4 result at TB diagnosis (aOR 0.16, CI 0.08-0.33), and lack of HIV testing before TB treatment (aOR 0.32, CI 0.18-0.59) significantly predicted lack of antiretroviral therapy. Our findings highlight the importance of HIV counseling and testing, as well as the need for strengthening pre-antiretroviral therapy care among people living with HIV within the study site 
province. ${ }^{38}$ It also reflects the interface between the universal coverage system for antiretroviral therapy and the burden of TB and HIV among non-Thai residents in Chiang Rai. A more integrated approach to TB and HIV control may be able to overcome these barriers.

\section{TB care}

Diagnosis of TB is difficult in HIV-infected patients. In the present study, a definitive diagnosis with microbiological evidence was documented in only a third of cases (Table 1). The mycobacterial laboratory in the high TB-prevalent setting of Chiang Rai was overloaded with hundreds of sputum samples but had a limited number of technicians. ${ }^{39}$ This might have caused delayed diagnosis and treatment. Late diagnosis of TB is likely to result in rapid deterioration of the immunological status of HIV-infected patients, leading to death shortly thereafter.

Moreover, routine microscopy and culture are not sensitive enough to detect active TB cases among people living with HIV. A diagnostic algorithm for diagnosis and screening of TB in HIV was developed by previous researchers in Thailand. ${ }^{40}$ Combined use of a highly sensitive screening algorithm as well as a rapid confirmatory diagnostic tool, such as Xpert MTB/RIF, is necessary. ${ }^{41}$ Further, the setting up of a diagnostic trial in Chiang Rai for early detection of TB is mandatory.

A multicenter study in Thailand has reported an increased risk of death in HIV-infected patients due to extrapulmonary $\mathrm{TB}$, particularly meningeal $\mathrm{TB} .{ }^{24}$ In our study, we found a large proportion $(40 \%)$ of extrapulmonary $\mathrm{TB}$, a third of which was TB meningitis. There is limited research evidence concerning the treatment of HIV-associated extra-pulmonary $\mathrm{TB}$, although a recent trial in Vietnam reported poor survival rates of PLWH suffering from TB meningitis, despite early ART and TB therapy. ${ }^{26}$ Research and clinical trials are still necessary, especially in settings experiencing a high prevalence of TB and HIV.

\section{Limitation}

This study was a retrospective death review study based on existing data. The validity and reliability of reconfirmed causes of death relied on the quality of the primary data recorded by certifying physicians. In the developing country setting, it is difficult to discern details concerning the immediate cause of death from death certificates and mortality statistics. Thirty of the cases were excluded because they lacked sufficient medical detail. The cause of death was unclear in $16 \%$ of the cases. Moreover, we could not obtain the required information about concomitant diseases, such as hepatitis B and C, which are prevalent in Thailand. Important laboratory markers such as liver enzymes were also lacking. Furthermore, our study sample selectively comprised deceased patients, thus interpretation of the association and predictors should be cautiously based on the study domain.

\section{Conclusion}

Despite its limitations, current study has given an overview of and an insight into mortality in patients with HIV-associated TB in Chiang Rai. It has also put into context the nature of TB-HIV care and the significant challenges it faces. These findings may provide the clues for medical care and future research to increase the life-expectancy of PLWH infected with TB.

\section{Acknowledgments}

Hutsaya Tantipong is gratefully acknowledged by the authors for her efforts in reviewing patient records. Thittaya Kulprayong, Maitri Oongern, Oranuch Nampaisan, the staff members of the TB/HIV Research Project, and the staff members of the TB clinic, HIV clinic, and laboratory of $\mathrm{CPH}$ are also acknowledged. Richard Lawrence Mann is acknowledged for refining the English language in this paper. The authors are grateful to Nobukatsu Ishikawa and Akira Shimochi, Research Institute of Tuberculosis, Japan Anti-Tuberculosis Association, for their kind and unwavering support.

\section{Authors' contribution}

PK and SM perceived the primary idea. PK, KM, SM, NY designed the study and collected the data. MNA designed the analysis. SM, NY and MNA analyzed the data.

MNA and all authors interpreted the result. MNA wrote the manuscript. MNA and PK finalized the article. All authors read and confirmed the article.

\section{Disclosure}

PK was in the review team that confirmed the causes of death. No other conflicts of interest are declared.

\section{References}

1. World Health Organiztion TB/HIV fact sheets. 2011. Available from: http://www.who.int/tb/publications/TBHIV_Facts_for_2011.pdf. Accessed June 4, 2012.

2. Cain KP, Anekthananon T, Burapat C, et al. Causes of death in HIVinfected persons who have tuberculosis, Thailand. Emerg Infect Dis. 2009;15(2):258-264.

3. Krentz H, Kliewer G, Gill M. Changing mortality rates and causes of death for HIV-infected individuals living in Southern Alberta, Canada from 1984 to 2003. HIV Med. 2005;6(2):99-106. 
4. Palella FJJ, Baker RK, Moorman AC, et al. Mortality in the highly active antiretroviral therapy era: changing causes of death and disease in the HIV Outpatient Study. J Acquir Immune Defic Syndr. 2006;43(1):27-34.

5. Palella FJ, Delaney KM, Moorman AC, et al. Declining morbidity and mortality among patients with advanced human immunodeficiency virus infection. $N$ Engl J Med. 1998;338(13):853-860.

6. Saraceni V, King BS, Cavalcante SC, et al. Tuberculosis as primary cause of death among AIDS cases in Rio de Janeiro, Brazil. Int JTuberc Lung Dis. 2008;12(7):769-772.

7. Straetemans M, Bierrenbach AL, Nagelkerke N, Glaziou P, van der Werf MJ. The effect of tuberculosis on mortality in HIV positive people: a meta-analysis. PLoS One. 2010;5(12):e15241.

8. Kyeyune R, den Boon S, Cattamanchi A, et al. Causes of early mortality in HIV-infected TB suspects in an East African referral hospital J Acquir Immune Defic Syndr. 2010;55(4):446-450.

9. Au-Yeung C, Kanters S, Ding E, et al. Tuberculosis mortality in HIVinfected individuals: a cross-national systematic assessment. Clin Epidemiol. 2011;3:21-29.

10. World Health Organization. TB/HIV Facts 2010. Available from: http:// www.who.int/tb/challenges/hiv/factsheet_hivtb_2010.pdf. Accessed November 4, 2011.

11. World Health Organization. Global Tuberculosis Control 2010. Available from: http://www.who.int/tb/features_archive/global_report2010_ launch_11nov10/en/index.html. Accessed August 12, 2012.

12. World Health Organization. South-East Asia Regional Office Tuberculosis Control in South-East Asia Region 2012. Available from: http://www.searo.who.int/LinkFiles/Tuberculosis_WHO-TB-Report2012.pdf. Accessed April 24, 2012.

13. US Global Health Policy. TB: HIV prevalence incident TB cases 2009. Available from: http://www.globalhealthfacts.org/topic.jsp?i=26 Accessed August 12, 2012.

14. World Health Organization. Tuberculosis country profiles 2012. Available from: http://www.who.int/tb/country/data/profiles/en/index. html. Accessed April 24, 2012.

15. Moolphate S. TB HIV situation in Chiang Rai province. Report presented at the 14th TB HIV Annual Meeting, January 13, 2011, Chiang Rai, Thailand.

16. Ansari NA, Kombe AH, Kenyon TA, et al. Pathology and causes of death in a group of 128 predominantly HIV-positive patients in Botswana, 1997-1998. Int J Tuberc Lung Dis. 2002;6(1):55-63.

17. Mankhatitham W, Lueangniyomkul A, Manosuthi W. Hepatotoxicity in patients co-infected with tuberculosis and HIV-1 while receiving nonnucleoside reverse transcriptase inhibitor-based antiretroviral therapy and rifampicin-containing anti-tuberculosis regimen. Southeast Asian J Trop Med Public Health. 2011;42(3):651-658.

18. World Health Organization. Treatment of Tuberculosis: Guidelines for National Programmes. 4th ed. Geneva, Switzerland: World Health Organization; 2009.

19. Department of Disease Control Ministry of Public Health. Thailand National Antiretroviral Treatment Guideline (2006/2007) 2007. Available from: http://www.gfaidscare.com/doc/Guideline $\% 20$ English\%20Part_one[1].pdf. Accessed August 12, 2012.

20. World Health Organization Stop TB Department. Treatment of tuberculosis: guidelines for national programmes. 4th ed. Available from: http://www.who.int/tb/publications/tb_treatmentguidelines/en/ index.html. Accessed June 27, 2012.

21. Akksilp S, Karnkawinpong O, Wattanaamornkiat W, et al. Antiretroviral therapy during tuberculosis treatment and marked reduction in death rate of HIV-infected patients, Thailand. Emerg Infect Dis. 2007;13(7):1001-1007.

22. Sanguanwongse NMD, Cain KPMD, Suriya PMS, et al. Antiretroviral therapy for HIV-infected tuberculosis patients saves lives but needs to be used more frequently in Thailand. J Acquir Immune Defic Syndr. 2008;48(2):181-189.
23. Varma J, Nateniyom S, Akksilp S, et al. HIV care and treatment factors associated with improved survival during TB treatment in Thailand: an observational study. BMC Infect Dis. 2009;9(1):42.

24. Kingkaew N, Sangtong B, Amnuaiphon W, et al. HIV-associated extrapulmonary tuberculosis in Thailand: epidemiology and risk factors for death. Int J Infect Dis. 2009;13(6):722-729.

25. Nissapatorn V. Lessons learned about opportunistic infections in southeast Asia. Southeast Asian J Trop Med Public Health. 2008;39(4): 625-641.

26. Torok ME, Yen NT, Chau TT, et al. Timing of initiation of antiretroviral therapy in human immunodeficiency virus (HIV)-associated tuberculous meningitis. Clin Infect Dis. 2011;52(11):1374-1383.

27. Tansuphasawadikul S, Saito W, Kim J, et al. Outcomes in HIV-infected patients on antiretroviral therapy with tuberculosis. Southeast Asian J Trop Med Public Health. 2007;38(6):1053-1060.

28. Moolphate S, Aung MN, Nampaisan O, et al. Time of highest tuberculosis death risk and associated factors: an observation of 12 years in northern Thailand. Int J Gen Med. 2011;4:181-190.

29. Gutierrez EB, Zanetta DM, Saldiva PH, Capelozzi VL. Autopsy-proven determinants of death in HIV-infected patients treated for pulmonary tuberculosis in Sao Paulo, Brazil. Pathol Res Pract. 2002;198(5): 339-346.

30. Harris JR, Lindsley MD, Henchaichon S, et al. High prevalence of cryptococcal infection among HIV-infected patients hospitalized with pneumonia in Thailand. Clin Infect Dis. 2012;54(5):e43-e50.

31. World Health Organization. Antiretroviral therapy for HIV infection in adults and adolescents; recommendations for a public health approach, 2010 revision. Available from: http://www.who.int/hiv/pub/arv/ adult2010/en/index.html. Accessed August 12, 2012.

32. Sungkanuparph S, Anekthananon T, Hiransuthikul N, et al. Guidelines for antiretroviral therapy in HIV-1 infected adults and adolescents: the recommendations of the Thai AIDS Society (TAS) 2008. J Med Assoc Thai. 2008;91(12):1925-1935.

33. Sungkanuparph S, Techasathit W, Utaipiboon C, et al. Thai national guidelines for antiretroviral therapy in HIV-1 infected adults and adolescents 2010. Asian Biomed (Res Rev News). 2010;4(4):515-528.

34. Abdool Karim SS, Naidoo K, Grobler A, et al. Integration of antiretroviral therapy with tuberculosis treatment. $N$ Engl $\mathrm{J} \mathrm{Med}$. 2011;365(16):1492-1501.

35. Havlir DV, Kendall MA, Ive P, et al. Timing of antiretroviral therapy for HIV-1 infection and tuberculosis. N Engl J Med. 2011;365(16): 1482-1491.

36. Blanc F-X, Sok T, Laureillard D, et al. Earlier versus later start of antiretroviral therapy in HIV-infected adults with tuberculosis. $N$ Engl J Med. 2011;365(16):1471-1481.

37. Lawn SD, Wood R. Timing of antiretroviral therapy for HIV-1associated tuberculosis. N Engl J Med. 2012;366(5):474-476.

38. Larson BA, Brennan A, McNamara L, et al. Early loss to follow up after enrolment in pre-ART care at a large public clinic in Johannesburg, South Africa. Trop Med Int Health. 2010;15 Suppl 1: 43-47.

39. Chaiyasirinroje B, Aung MN, Moolphate S, et al. Prospective evaluation of simply modified MODS assay: an effective tool for TB diagnosis and detection of MDR-TB. Infect Drug Resist. 2012;5: 79-86.

40. Cain KP, McCarthy KD, Heilig CM, et al. An algorithm for tuberculosis screening and diagnosis in people with HIV. NEngl J Med. 2010;362(8):707-716.

41. Van Rie A, Page-Shipp L, Scott L, Sanne I, Stevens W. Xpert(R) $\mathrm{MTB} / \mathrm{RIF}$ for point-of-care diagnosis of TB in high-HIV burden, resource-limited countries: hype or hope? Expert Rev Mol Diagn. 2010;10(7):937-946. 


\section{Publish your work in this journal}

HIV/AIDS - Research and Palliative Care is an international, peerreviewed open-access journal focusing on advances in research in HIV, its clinical progression and management options including antiviral treatment, palliative care and public healthcare policies to control viral spread. The journal welcomes original research, basic science,

clinical \& epidemiological studies, reviews \& evaluations, expert opinion \& commentary, case reports \& extended reports. The manuscript management system is completely online and includes a very quick and fair peer-review system. Visit http://www.dovepress.com/ testimonials.php to read real quotes from published authors.

Submit your manuscript here: http://www.dovepress.com/hivaids---research-and-palliative-care-journal 